\title{
Robotic assisted minimally invasive esophagectomy (RAMIE): the University of Pittsburgh Medical Center initial experience
}

\author{
Olugbenga T. Okusanya ${ }^{*}$, Inderpal S. Sarkaria ${ }^{*}$, Nicholas R. Hess, Katie S. Nason, Manuel Villa Sanchez, \\ Ryan M. Levy, Arjun Pennathur, James D. Luketich \\ Department of Cardiothoracic Surgery, University of Pittsburgh School of Medicine and the University of Pittsburgh Medical Center, Pittsburgh, \\ PA, USA \\ *These authors contributed equally to this work. \\ Correspondence to: Inderpal S. Sarkaria, MD, FACS. Department of Cardiothoracic Surgery, Division of Thoracic Surgery, University of Pittsburgh \\ Medical Center, Shadyside Medical Building, Suite 715.27, Pittsburgh, PA 15232, USA. Email: sarkariais@upmc.edu.
}

Submitted Feb 14, 2017. Accepted for publication Mar 09, 2017.

doi: $10.21037 / \mathrm{acs} .2017 .03 .12$

View this article at: http://dx.doi.org/10.21037/acs.2017.03.12

\section{Introduction}

Esophagectomy is the mainstay of therapy in appropriately selected patients with resectable malignant esophageal disease (1). However, esophagectomy remains a technically challenging procedure that has the potential for significant postoperative morbidity and mortality $(2,3)$.

Over the last 20 years, minimally invasive esophagectomy (MIE) has become increasingly adopted as a means to potentially decrease the perioperative morbidity of these operations. At the University of Pittsburgh Medical Center (UPMC), MIE has been shown to be a safe and effective procedure with broad applicability and equivalent oncologic outcomes (4-6).

More recently, robotic assisted approaches to these operations have been increasingly described with early series reporting varying techniques and outcomes (7-12). Larger single institution series, including from Memorial Sloan Kettering Cancer Center and others, have reported systematic approaches in the development of a robotic assisted minimally invasive esophagectomy (RAMIE) program yielding excellent outcomes with increasing proficiency over the course of the learning curve (13-15). The primary purported benefit of the robotic assisted approach largely centers around the markedly increased control over the conduct of the operation afforded to the operator over open or alternative minimally invasive operations. The primary purpose of this study is to report the initial experience with RAMIE at the UPMC, a high volume teaching program with extensive experience in minimally invasive esophageal operations.

\section{Methods \\ Patient selection}

Between 2014 and 2016 patients seen for consideration of MIE were also considered for RAMIE. No specific selection criteria were specified, and patients considered appropriate for MIE were also considered appropriate for RAMIE. All patients underwent preoperative staging and evaluation including a full history and exam, esophagogastroduodenoscopy (EGD) with biopsy, fluorodeoxyglucose-18 positron emission tomography, computed tomography of the chest abdomen and pelvis and endoscopic ultrasound. Patients with suspected T3 or node positive tumors were referred for neoadjuvant chemotherapy or chemoradiation therapy and reevaluated for surgery following induction treatment.

The co-first author (I.S.S.), an experienced robotic thoracic surgeon including expertise in RAMIE, acted as primary or co-surgeon on the robotic console for all cases. The majority of cases were also performed with the senior author (J.D.L.), a highly experienced minimally invasive and esophageal senior surgeon, as co-surgeon. All cases were also assisted by surgical trainees who took part in various aspects of the case at the teaching console or bedside, as well as a single experienced physician assistant as the bedside operator. The same protocols used to manage the post-operative care of the MIE patients was used in the care 
of the RAMIE patients.

\section{Data collection}

This study was granted a waiver from the institutional review board (IRB) for retrospective study and review. Patient characteristics and outcomes were collected and recorded in prospective fashion in accordance with an ongoing esophageal surgery database. Postoperative complications and long term follow up was collected prospectively and retrospectively by chart review. Complications were graded using the Clavien Dindo Grading Score (16).

\section{Operative technique}

\section{Abdominal approach}

Our approach to RAMIE has been previously described by the co-first author, and was largely adapted from the MIE approach originally described and developed at UPMC $(5,13)$. To summarize, EGD and bronchoscopy are performed at the beginning of every case. A midline $8 \mathrm{~mm}$ robotic port is placed at the level of the umbilicus. Three more $8 \mathrm{~mm}$ ports are placed in left and right mid clavicular line and at the left costal margins. A 5-mm non-robotic port is placed at the right costal margin through which a liver retractor is placed. A robotic bipolar forceps are used in the right midclavicular port, ultrasonic shears in the left midclavicular port and an atraumatic grasper in the leftmost costal port. An assistant $12 \mathrm{~mm}$ non-robotic port is placed in the right para umbilical position, as well as a second $5 \mathrm{~mm}$ assistant port further lateral in the same para umbilical line.

The dissection is generally begun with division of the lesser omentum, initial assessment and mobilization of the crura and esophageal hiatus, and exposure of the left gastric vascular pedicle. Complete celiac axis lymphadenectomy is performed, dissecting and sweeping all celiac, splenic and retrogastric lymphatic bearing tissues up along the vascular pedicle for later en bloc removal with the specimen. The left gastric and short gastric vessels are divided and the gastroepiploic arcade preserved in its entirety during gastric mobilization. Near infrared fluorescence imaging with indocyanine green may be utilized to clearly identify and preserve the gastroepiploic arcade to its termination point (15). In the setting of previous induction chemoradiation therapy, an omental flap based off of 2-3 omental perforating arteries may be harvested for later reinforcement of the gastroesophageal anastomosis. Complete gastric mobilization from the hiatus to the pylorus is performed. The gastric conduit is created with sequential applications of the endogastrointestinal stapler. The conduit is secured to the specimen for later traverse into the chest in proper orientation. The omental flap, if created, should be secured to the tip of the conduit to simplify transit into the chest as well.

A pyloroplasty is routinely performed in the majority of cases. The pylorus is open longitudinally with the ultrasonic shears and closed transversely with robotic suturing using interrupted sutures in a Heinicke-Mickulicz fashion. A feeding jejunostomy is placed and the abdominal portion concluded. The specimen is secured to the conduit and the abdominal portion is concluded.

\section{Thoracic approach}

The patient is placed in standard left lateral decubitus position. $\mathrm{CO}_{2}$ insufflation is initiated with an entry needle just below the tip of the scapula. Eight mm robotic ports are placed at the eighth intercostal space at the posterior axillary line, the third intercostal space in the mid to posterior axillary, fifth intercostal place into the mid axillary line, and at the ninth intercostal space approximately in line with the tip of the scapula. An assistant non-robotic port is placed at the site of the diaphragmatic insertion. Complete circumferential esophageal mobilization is performed from the level of the hiatus to the azygous vein with careful attention to harvest all periesophageal lymph node bearing tissues en bloc with the specimen. During dissection of the subcarinal lymph node packet, great care must be taken to avoid energy associated thermal injury to the membranous wall of the airways. Judicious use of both bipolar energy sources and non-energy dependent sharp and blunt dissection, and clear visualization and exposure of the dependent anatomy are critical to avoid these injuries which may result in esophageal/conduit airway fistulas, a known pitfall of MIE, robotic or otherwise (7,13-14). Additional mobilization of the esophagus towards the thoracic inlet is completed with careful attention to avoid traction or direct injury to the recurrent laryngeal nerve. The conduit is delivered into the chest and sutured to the diaphragm. The caudal to cranial deep dissection along the contralateral pleura and left mainstem bronchus is completed with lateral retraction of the specimen once divided from the conduit.

The esophagus is divided approximately $2-3$ centimeters above the azygos vein, although more proximal division may be performed dependent on the margins necessary. 
A 4-5 cm access incision in made through the operator's "left" hand robotic working port to deliver the specimen out of the chest. A robotically placed running "baseball" suture is placed around the opening of the divided proximal esophagus, and the anvil of the $28 \mathrm{~mm}$ end to end anastomotic (EEA) stapler is inserted and secured. An additional reinforcing superficial purse string suture is placed to ensure tissue apposition around the stem of the anvil during deployment of the stapler. The EEA stapler is introduced through a gastrotomy site created in the proximal conduit tip, and the spike brought out through the lateral wall of the conduit ideally just above the level of the vascular arcade insertion. The stapler is then docked to the anvil and fired, creating the anastomosis, and the redundant conduit resected. If an omental flap has been harvested, it is loosely secured around the newly created anastomosis at this time. A drain is left posterior to the conduit and a chest tube is left in the right pleural space.

Per our post-operative pathways for non-complicated cases, patients are generally admitted to intensive care unit (ICU) on the day of surgery and discharged to step-down on postoperative day 1 or 2 . Tube feeding is initiated on day 2. A barium swallow is performed after removal of the nasogastric tube on postoperative day 4-5 and a liquid diet initiated. Patients are discharged with the perianastomotic drain which is removed at the first postoperative visit if no evidence of anastomotic leak is observed.

\section{Results}

\section{Patient demographics}

Patient demographics and tumor characteristics are summarized in Table 1. Twenty-five patients underwent RAMIE from June of 2014 until October of 2016. The mean age of these patients was 67 years old with a range from 39 to 84 years. Eighty percent of these patients were male. Fourteen $(56 \%)$ of these patient received neoadjuvant chemoradiation while four (16\%) received neoadjuvant chemotherapy. Seven (28\%) patients underwent RAMIE without previous neoadjuvant therapy.

\section{Preoperative tumor characteristics}

Eighteen (72\%) of the patients underwent RAMIE for esophageal adenocarcinoma (ACC), six (24\%) for squamous cell carcinoma (SCC), and one (4\%) for adenosquamous carcinoma. The majority of patients presented with stage
Table 1 Patient demographics and tumor characteristics in 25 patients undergoing RAMIE

\begin{tabular}{lc}
\hline Variable & Value (range or \%) \\
\hline Median age & 67 (range, 39-84) \\
Male gender & $20[80]$ \\
Induction therapy & \\
None & $7[28]$ \\
Chemotherapy & $4[16]$ \\
Chemotherapy and radiation & $14[56]$ \\
ASA risk class & \\
2 & $5[20]$ \\
3 & $20[80]$ \\
Approach & \\
Ivor Lewis & $23[92]$ \\
McKeown & $2[8]$
\end{tabular}

Histology

Adenocarcinoma 18 [72]

Squamous cell carcinoma

Adenosquamous carcinoma

Clinical stage

IA

IB

\section{Pathologic stage}

0 (complete response)

IB

IIA

2 [8]

IIB

6 [24]

IIIA

4 [16]

IIIB

4 [16]

IIIC

2 [8]

Table 1 (continued) 


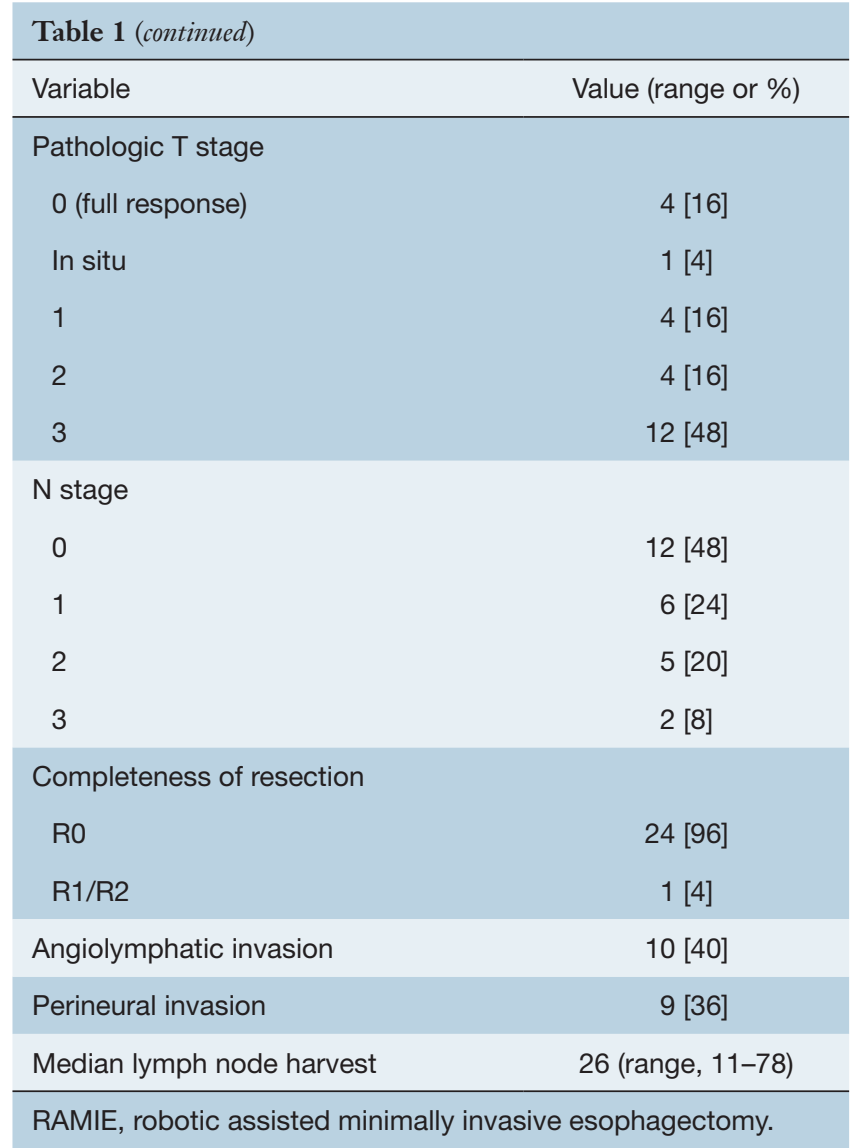

IIIA or IIIB disease (32\% and $24 \%$ respectively).

\section{Operative variables}

Perioperative outcomes and complications are summarized in Table 2. The median operative time (skin incision to skin closure) was 661 minutes with a range of 503 to 902 minutes. Median estimated blood loss was $250 \mathrm{cc}$. A mean number of 26 lymph nodes were harvested with a range of 11 to 78 . There were 4 total conversions with 2 (8\%) unplanned conversions. One conversion was to open laparotomy due to extensive intra-abdominal adhesions and the other to non-robotic minimally invasive surgery for routine thoracoscopic creation of the anastomosis.

\section{Postoperative outcomes}

The median length of stay was 8 days with a median
Table 2 Perioperative outcomes and complications in 25 patients undergoing RAMIE

\begin{tabular}{|c|c|}
\hline Variable & Value (range or \%) \\
\hline Mean operative time (minutes) & 661 (range, 503-902) \\
\hline Median estimated blood loss (mL) & 250 (range, 50-700) \\
\hline \multicolumn{2}{|l|}{ Conversions (unplanned) } \\
\hline To non-robotic MIS & $1[4]$ \\
\hline To open & $1[4]$ \\
\hline Median ICU length of stay (days) & 2 (range, 1-10) \\
\hline Median hospital length of stay (days) & 8 (range, 6-20) \\
\hline \multicolumn{2}{|l|}{ Complications (Clavien Dindo) } \\
\hline Class I & 3 \\
\hline Postoperative ileus & 1 \\
\hline Urinary retention & 1 \\
\hline Incisional cellulitis & 1 \\
\hline Class II & 16 \\
\hline Atrial fibrillation & 6 \\
\hline Pneumonia & 3 \\
\hline $\begin{array}{l}\text { Pleural effusion requiring catheter } \\
\text { drainage }\end{array}$ & 2 \\
\hline SVT & 1 \\
\hline Hyponatremia & 1 \\
\hline Delirium & 1 \\
\hline Decubitus ulcer & 1 \\
\hline Chyle leak & 1 \\
\hline Class IIla & 3 \\
\hline $\begin{array}{l}\text { Respiratory failure requiring ICU } \\
\text { readmission }\end{array}$ & 1 \\
\hline Class IIIb & 1 \\
\hline $\begin{array}{l}\text { Respiratory failure requiring } \\
\text { tracheostomy }\end{array}$ & 1 \\
\hline Class IV & 1 \\
\hline Endocarditis & 1 \\
\hline Anastomotic leak $\geq$ grade 2 & $1[4]$ \\
\hline 90 day mortality & 0 \\
\hline Follow up time (months) & 9.2 (range, 0.9-27.3) \\
\hline
\end{tabular}

RAMIE, robotic assisted minimally invasive esophagectomy. 


\begin{tabular}{|c|c|c|c|}
\hline Patient number & 1,011 & 21 & 25 \\
\hline Age, median & 64 & 62 & 67 \\
\hline \multicolumn{4}{|l|}{ Histology, n [\%] } \\
\hline Adenocarcinoma & $727[76]$ & $18[85]$ & 18 [72] \\
\hline Other & $179[13]$ & $1[5]$ & $1[4]$ \\
\hline Median operative time, minutes & NR & 556 & 661 \\
\hline Median estimated blood loss, $\mathrm{mL}$ & NR & 307 & 250 \\
\hline Median hospital length of stay, days & 8 & 10 & 8 \\
\hline Anastomotic leak, $\mathrm{n}[\%]$ & $26[5]$ & $2(9.5)$ & $1[4]$ \\
\hline 30-day mortality, n [\%] & $17[1.7]$ & $0[0]$ & $0[0]$ \\
\hline
\end{tabular}

ICU length of stay of 2 days. Eight (32\%) patients had uncomplicated hospital stays. One patient (4\%) suffered a grade 2 or greater anastomotic leak. All other complications are summarized in Table 2.

There were no deaths within the 30- or 90-day postoperative period. No patients were lost to follow up. The mean follow up time was 9 months with a range of one to 27 months. In 24 (96\%) patients, a complete resection with microscopically negative margins (R0) was obtained. Four patients (16\%) had complete pathologic responses after neoadjuvant therapy.

\section{Discussion}

This study represents our initial experience with RAMIE at the UPMC, and suggests that the safe introduction of these procedures can be accomplished with excellent outcomes in the setting of a high volume esophageal practice with surgeons already proficient in MIE and robotic surgery. Compared to a large series of over 1,000 patients undergoing MIE at the UPMC, RAMIE and MIE patients had similar 30 day mortality ( $0 \%$ vs. $2.8 \%$ ), clinically significant anastomotic leak (4\% vs. 5\%), median lymph nodes harvested (27vs. 21), conversion rates ( $8 \%$ vs. $5 \%$ ), and R0 resection (96\% vs. 98\%) (Table 3) (4). RAMIE operative times were greater and likely represent an early learning curve phenomenon, similar to that observed early and subsequent series from Memorial Sloan Kettering Cancer Center reported by the current co-first author (I.S.S.) $(7,14)$. Interestingly, this learning curve phenomenon did not appear to be attenuated with the presence of an experienced RAMIE surgeon, suggesting an institutional learning curve, at least for time, independent of the operating surgeon's alone. Other elements of the learning curve in the current series were decreased compared to the early MSKCC series, including rates of conversion ( $8 \%$ vs. $42 \%)$ and early rates of anastomotic leak (4\% vs. 14\%). Of note, there were no enteric-airway fistulas in this series, potentially representing the extensive accumulated previous experience of the senior surgeons in robotic.

There are several potential advantages to the robotic platform in these procedures. Tissue dissection in areas such as the hiatus and mediastinum, especially in patients with marked response to neoadjuvant therapy, may be facilitated by the superior optics and visualization, as well as instrumentation with multiple degrees of freedom, afforded by the robotic platform. The addition of a central camera, as well as an additional "assistant" arm, both under direct control of the surgeon, decrease the reliance on surgical assistants and greatly elevate the surgeon's control over the 
conduct of the operation. Technically challenging portions of the MIE operation with long learning curves, such as pyloroplasty and creation of the stapled anastomosis, may be greatly facilitated with robotic suturing capabilities. While direct clinical benefit to the patient may be difficult to quantify, the benefits to the surgeon in terms of ease and simplification of self-orchestrated operative performance, and potential decrease in chronic work-related trauma and injuries, particularly involving long and complex operations, may be significant. As a caveat beyond the scope of this study, the financial and cost implications of these procedures are not currently well delineated within a large university practice with multiple surgical service lines utilizing robotic platforms. These potential costs in contrast to the potential benefits merit additional study to quantitatively characterize.

While our early RAMIE data is naturally limited by its relatively low volume of cases, the initial results are encouraging and do not suggest a compromise in surgical and early oncological outcomes with inception of the program within a high volume esophageal center of excellence with expertise in MIE. These institutional traits may represent a "best-case" scenario for development of a RAMIE program, but also represent a limitation of the study in that it is not clear what the applicability of these findings may be to other centers with less a priori experience. Regardless of practice specific background, much care and consideration must be taken to balance the needs of training surgeons in these complex robotic procedures without subjecting patients to unnecessary or undue risk. Preclinical observation of cases, simulation and stylized curriculum based training at established robotic RAMIE programs, case proctorship, and careful and graded accumulation of RAMIE experience with a priority on maintaining patient safety and outcomes may all help promote successful navigation of the learning curve without recapitulation of recognized and preventable procedural pitfalls, morbidity, and mortality.

\section{Acknowledgements}

None.

\section{Footnote}

Conflicts of Interest: The authors have no conflicts of interest to declare.

\section{References}

1. Abrams JA, Buono DL, Strauss J, et al. Esophagectomy compared with chemoradiation for early stage esophageal cancer in the elderly. Cancer 2009;115:4924-33.

2. Briel JW, Tamhankar AP, Hagen JA, et al. Prevalence and risk factors for ischemia, leak, and stricture of esophageal anastomosis: gastric pull-up versus colon interposition. J Am Coll Surg 2004;198:536-41; discussion 541-2.

3. Raymond D. Complications of esophagectomy. Surg Clin North Am 2012;92:1299-313.

4. Luketich JD, Pennathur A, Awais O, et al. Outcomes after minimally invasive esophagectomy: review of over 1000 patients. Ann Surg 2012;256:95-103.

5. Pennathur A, Awais O, Luketich JD. Technique of minimally invasive Ivor Lewis esophagectomy. Ann Thorac Surg 2010;89:S2159-62.

6. Luketich JD, Pennathur A, Franchetti Y, et al. Minimally invasive esophagectomy: results of a prospective phase II multicenter trial-the eastern cooperative oncology group (E2202) study. Ann Surg 2015;261:702-7.

7. Sarkaria IS, Rizk NP, Finley DJ, et al. Combined thoracoscopic and laparoscopic robotic-assisted minimally invasive esophagectomy using a four-arm platform: experience, technique and cautions during early procedure development. Eur J Cardiothorac Surg 2013;43:e107-15.

8. Ruurda JP, Draaisma WA, van Hillegersberg R, et al. Robot-assisted endoscopic surgery: a four-year singlecenter experience. Dig Surg 2005;22:313-20.

9. van Hillegersberg R, Boone J, Draaisma WA, et al. First experience with robot-assisted thoracoscopic esophagolymphadenectomy for esophageal cancer. Surg Endosc 2006;20:1435-9.

10. Anderson C, Hellan M, Kernstine K, et al. Robotic surgery for gastrointestinal malignancies. Int J Med Robot 2007;3:297-300.

11. Galvani CA, Gorodner MV, Moser F, et al. Robotically assisted laparoscopic transhiatal esophagectomy. Surg Endosc 2008;22:188-95.

12. Cerfolio RJ, Wei B, Hawn MT, et al. Robotic Esophagectomy for Cancer: Early Results and Lessons Learned. Semin Thorac Cardiovasc Surg 2016;28:160-9.

13. Sarkaria IS, Rizk NP. Robotic-assisted minimally invasive esophagectomy: the Ivor Lewis approach. Thorac Surg Clin 2014;24:211-22, vii.

14. Sarkaria IS, Rizk NP, Grosser R, et al. Attaining 
Proficiency in Robotic-Assisted Minimally Invasive Esophagectomy While Maximizing Safety During Procedure Development. Innovations (Phila) 2016;11:268-73.

15. van der Sluis PC, Ruurda JP, Verhage RJ, et al. Oncologic Long-Term Results of Robot-Assisted Minimally Invasive

Cite this article as: Okusanya OT, Sarkaria IS, Hess NR, Nason KS, Sanchez MV, Levy RM, Pennathur A, Luketich JD. Robotic assisted minimally invasive esophagectomy (RAMIE): the University of Pittsburgh Medical Center initial experience. Ann Cardiothorac Surg 2017;6(2):179-185. doi: 10.21037/ acs.2017.03.12
Thoraco-Laparoscopic Esophagectomy with Two-Field Lymphadenectomy for Esophageal Cancer. Ann Surg Oncol 2015;22 Suppl 3:S1350-6.

16. Clavien PA, Barkun J, de Oliveira ML, et al. The ClavienDindo classification of surgical complications: five-year experience. Ann Surg 2009;250:187-96. 